
\section{BMJ Quality}

\title{
Red eyes and red-flags: improving ophthalmic assessment and referral in primary care
}

\author{
Caroline Kilduff, Charis Lois
}

To cite: Kilduff C, Lois $\mathrm{C}$. Red eyes and red-flags: improving ophthalmic assessment and referral in primary care. BMJ Quality Improvement Reports 2016;5:u211608.w4680. doi:10.1136/bmjquality. u211608.w4680

Received 14 March 2016 Revised 28 April 2016

\section{CrossMark}

\section{St Mary's Hospital, UK}

Correspondence to Caroline Kilduff caroline. kilduff@imperial.nhs.uk

\section{ABSTRACT}

Up to five percent of primary care consultations are eye-related, yet $96 \%$ of General Practitioners (GPS) do not undergo postgraduate ophthalmology training. Most do not feel assured performing eye assessments. Some red eye conditions can become sight threatening, and often exhibit red-flag features. These features include moderate pain, photophobia, reduced visual acuity (VA), eye-trauma, or unilateral marked redness. The aim of this project was to improve primary care assessment and referral of patients presenting with red-flag features based on the NICE 'Red Eye' Clinical Knowledge Summary recommendations.

Data was collected retrospectively from 139 red eye consultations. A practice meeting highlighted poor awareness of red-flag features, low confidence levels in eye assessments, and time-constraints during appointments. Interventions were based on feedback from staff. These included a primary care teaching session on red-flag features, a VA measurement tutorial, and provision of a red eye toolkit, including VA equipment, to each consultation room.

At baseline, each patient had on average 0.9 red-flag features assessed. Only $36.0 \%$ (9/25) of patients with red-flag features were appropriately referred to sameday ophthalmology services. Following two improvement cycles, a significant improvement was seen in almost every parameter. On average, each patient had 2.7 red-flag features assessed (vs 0.9, $p<0.001)$. VA was assessed in $55.6 \%$ of consultations (vs $7.9 \%, p<0.001$ ), pain was quantified in $81.5 \%$ (vs $20.9 \%, p=0.005)$, eye-trauma or foreign-body $(51.8 \%$ vs $8.6 \%, p<0.001)$, extent of redness was documented in $66.7 \%$ (vs $14.4 \%, p<0.001$ ). Only photophobia remained poorly assessed (18.5\% vs $14.4 \%, p=0.75)$. Following this, $75.0 \%(6 / 8)$ of patients were appropriately referred.

This project reflected the literature regarding low confidence and inexperience amongst GPs when faced with ophthalmic conditions. Improvements in education are required to ensure accurate assessments can be undertaken in a time-constrained environment.

\section{PROBLEM}

Between two and five percent of all presentations to general practice are ophthalmic in nature. ${ }^{1}$ Red eye is the descriptive term representing inflammation of the anterior segment resulting in vasodilation of the superficial blood vessels, ${ }^{2}$ and is the most common ocular presentation in general practice. ${ }^{3}$ In most cases, the cause is benign. However, sometimes a red eye may indicate a sight-threatening, or in extreme cases, a lifethreatening condition. ${ }^{4}$ According to the National Institute of Clinical Excellence (NICE), the benign conditions include bacterial, viral, and allergic conjunctivitis, episcleritis, and subconjunctival haemorrhage. These make up about $70 \%$ of the primary care red eye consultations, and are subsequently over-diagnosed. ${ }^{6}$ The more common sight-threatening causes of a red eye include keratitis, scleritis, acute glaucoma, foreign body trauma, chemical burns, and orbital cellulitis. Studies have demonstrated that accurate diagnosis of eye conditions is achieved between $16 \%$ and $36 \%$ of the time, and up to $12 \%$ of those misdiagnosed experience adverse outcomes as a result. ${ }^{7}$

In order to ensure that patients attending primary care services with a red eye are managed appropriately and safely, the NICE 'Red Eye' Clinical Knowledge Summary (CKS) specifies that the doctor must 'always document the history and findings of an eye examination (including negative findings). Pain, photophobia, visual acuity, and whether the condition is unilateral or bilateral, are the essential elements to be documented'. The clinician should refer the patient for same-day specialist assessment by an ophthalmologist if any red flag features are present. ${ }^{5}$

The aim of this project was to ensure that an assessment for red-flag features was performed in every patient presenting to their GP with a red eye, and those with red-flag features were appropriately referred to same-day ophthalmology services, as recommended by NICE in the 'Red Eye' CKS. ${ }^{5}$ The timeframe within which improvement was to be demonstrated was initially set at eight 
weeks. The setting was a primary care health centre, located in Tower Hamlets, serving a local population of over 13,000 patients.

\section{BACKGROUND}

After death, loss of vision is feared over any other longterm health condition. Sight loss has a negative impact on quality of life; a third of those who lose their vision develop depression, and two-thirds of working age adults registered as sight impaired are unemployed. ${ }^{8}$ The ability to accurately assess the eye is an essential skill that all doctors should possess, in order to ensure prompt and appropriate management of eye conditions, and prevent avoidable sight loss.

Benign and sight-threatening conditions can present very similarly. There are, however, five red flag features that indicate a potential sight-threatening condition. These are:

1. Moderate to severe eye pain

2. Photophobia

3. Marked redness of affected area

4. Reduced visual acuity

5. Foreign body or penetrating eye injury

Assessment of a red eye in the primary care setting should be tailored to exclude these features. ${ }^{5}$

General practice is often the first port of call for patients who develop a red eye, and the Royal College of General Practitioners (RCGP) chose eye health as a clinical priority from 2013-16. The college specified that the general practitioner (GP) should be able to 'examine, diagnose, and treat common eye conditions and know when to refer the patient to secondary care'. ${ }^{9}$ However, a survey study demonstrated that two-thirds of GPs admitted to both uncertainty and anxiety regarding assessment and management of eye conditions. Ten percent confessed to eye conditions 'scaring' them and most demonstrated reluctance to perform ocular assessments; seven percent of GPs in the survey admitted to never checking visual acuity, and one in five never testing visual fields. ${ }^{10}$ Although these investigations can be performed easily in primary care, there is a common misconception that an eye assessment cannot be done without specialist equipment. ${ }^{6}$

The RCGP stated that $50 \%$ of sight loss could be avoided through improved eye care and early detection of problems. ${ }^{9}$ Roy (2006) advised that the majority of red eye conditions can be managed in primary care, provided the clinician is able to differentiate between sightthreatening and benign conditions. ${ }^{11}$ However, when healthcare professionals lack experience in a subject area, their diagnostic reasoning becomes largely based on heuristics, leading to diagnostic error. ${ }^{12}$ Low confidence levels can impair clinical performance, resulting in inconsistent and sometimes illogical approaches to eye assessment and diagnosis. ${ }^{6}$

Only $3.7 \%$ of GP trainees undergo postgraduate ophthalmology training. The majority rely on their undergraduate knowledge of eye conditions when faced with an ocular presentation. Undergraduate ophthalmology training is currently suboptimal for this purpose; clinical placements are not compulsory, the average length of attachments are eight days, and ophthalmology teaching varies in content and depth between institutions. ${ }^{13}$ In one survey study, $80 \%$ of GPs requested protocols for assessing and managing common eye conditions, as well as informal teaching sessions on common eye conditions.

Clinicians will be faced with eye conditions, particularly red eye, in all areas of healthcare. Mahmood \& Narang (2008) highlighted the importance for emergency-care physicians to recognise red-flag features, and become confident in assessing and triaging red eye patients. ${ }^{14}$ Shields \& Sloane (1991) suggested that medical schools, and postgraduate primary care education facilities should emphasise the importance of knowledge regarding the common ocular presentations. ${ }^{15}$ Foster (2005) described the advantages of accurate primary care red eye assessments, not only to facilitate correct management, but to provide relief to secondary care, allowing resources to be focused on sight-threatening conditions. ${ }^{16}$

\section{BASELINE MEASUREMENT}

The initial data collection took place in February 2015. Baseline measurements were collected retrospectively from electronic records comprising 261 consultations at the Health Centre between 1st January 2013 and 1st January 2015. All consultations with codes including red eye, subconjunctival haemorrhage, and conjunctivitis (if the eye was documented as being red), were included if the patient was ten years or older at the date of consultation. Telephone consultations were excluded because the GP was unable to examine the eye. Those consultations coded conjunctivitis where the eye was not documented as being red were also excluded, as were patients under the age of ten years, as they would be less likely to comply with a visual acuity examination using the practice Snellen-chart. Data was therefore analysed from 139 consultations in total.

For each patient, it was observed whether each of the five red-flag features had been assessed and documented by the GP during the consultation. Documentation of the laterality of the symptoms was also evaluated. If a red-flag feature had been assessed, it was noted whether there was a positive finding. If the feature was present, it was determined whether the patient had been referred to same-day ophthalmology services. If a specific red-flag feature was not assessed, it was determined whether another red-flag feature had already been identified. Further data was collected and analysed for redness and pain to determine whether the extent of the redness or the severity of the pain had been quantified, as the qualification for these features to be a red-flag is dependent on the quantification. 
Data analysis was undertaken using the online calculator GraphPad (GraphPad Software, La Jolla California USA). Parametric data was analysed using the unpaired T-test. Non-parametric data was entered into a $2 \times 2$ contingency table and significance was calculated using a two-tailed Fisher's exact test.

Laterality of the affected eye was documented $100.0 \%$ of the time (139/139). This was due to an existing template on the consultation software which required the GP to enter laterality whenever an eye consultation was coded.

Only $7.9 \%(11 / 139)$ red eye patients had their visual acuity (VA) formally measured and an assessment of the presence ocular pain performed in $35.3 \%$ (49/139) of consultations. The severity of the pain was only quantified in $20.9 \%$ (29/139). Presence of a foreign body or suspicion of trauma was only assessed in $8.6 \%(12 / 139)$ of the consultations, and photophobia was only assessed $14.4 \%$ of the time $(20 / 139)$, yet of the patients who had a red-flag feature, photophobia was the feature identified in $40.0 \%(10 / 25)$.

Although the eye was documented to be red in $79.1 \%$ of consultations $(110 / 139)$, redness only fulfilled the criteria of a red-flag feature if it was considered to be marked redness. The extent of the redness was documented in only $14.4 \%(20 / 139)$ of assessments.

At baseline, none of patients presenting to the practice with a red eye had more than three red-flag features assessed and documented. In 41.7\% (58/139) no assessment was performed for any of the potential red-flag features. The mean number of red-flag features assessed per patient was 0.9 .

In the consultations where some red-flag features were assessed, 30.9\% (25/81) had a positive feature. Only $36.0 \%$ of these patients $(9 / 25)$ were appropriately referred to same-day ophthalmology services.

The baseline results demonstrated that the standards recommended by the NICE "Red Eye" CKS (2012), were not being met, and changes to practise were required in order to improve red eye assessments and referrals.

\section{DESIGN}

The baseline results demonstrated a general lack of awareness of the red-flag features, and in some cases, it was apparent that although the GP was addressing certain red-flag features, they demonstrated a lack of knowledge regarding appropriate assessment modalities.

The intervention was an interactive teaching session delivered to the staff at the health-centre. This was considered to be the most effective method of improving familiarity with ophthalmic examinations, and reinforcing the red-flag features. A teaching session would allow for immediate feedback, discussion on barriers to red-flag assessments, and maximise staff involvement in the intervention.

The session objectives included:

1. Common causes of red eye
2. A description of the sight-threatening conditions

3. Identification of red-flag features

4. How to assess each of the red-flag features

5. The appropriate referral pathway for red eye patients

6 . The results of the baseline data collection at the practice

The session was complete with two illustrated poster hand-outs; the first demonstrating the red-flag features (figure 1) and the second demonstrating a step-by-step guide to visual acuity assessment (figure 2). The poster aesthetics drew on influences from the design and advertising industry; which places huge emphasis on clear and simple visual communication to drive important messages to the service user.

Sustainability of the intervention was considered. The posters were distributed with the intention that they would be kept by the clinical staff as reminders of the red-flag features, referral pathway, and correct methodology for red-flag feature assessments.

\section{STRATEGY}

\section{Cycle 1:}

A meeting was scheduled with all staff at the practice. It was advertised by poster and via circular emails, and took place during a regular practice meeting time-slot in order to maximise attendance. During this meeting the

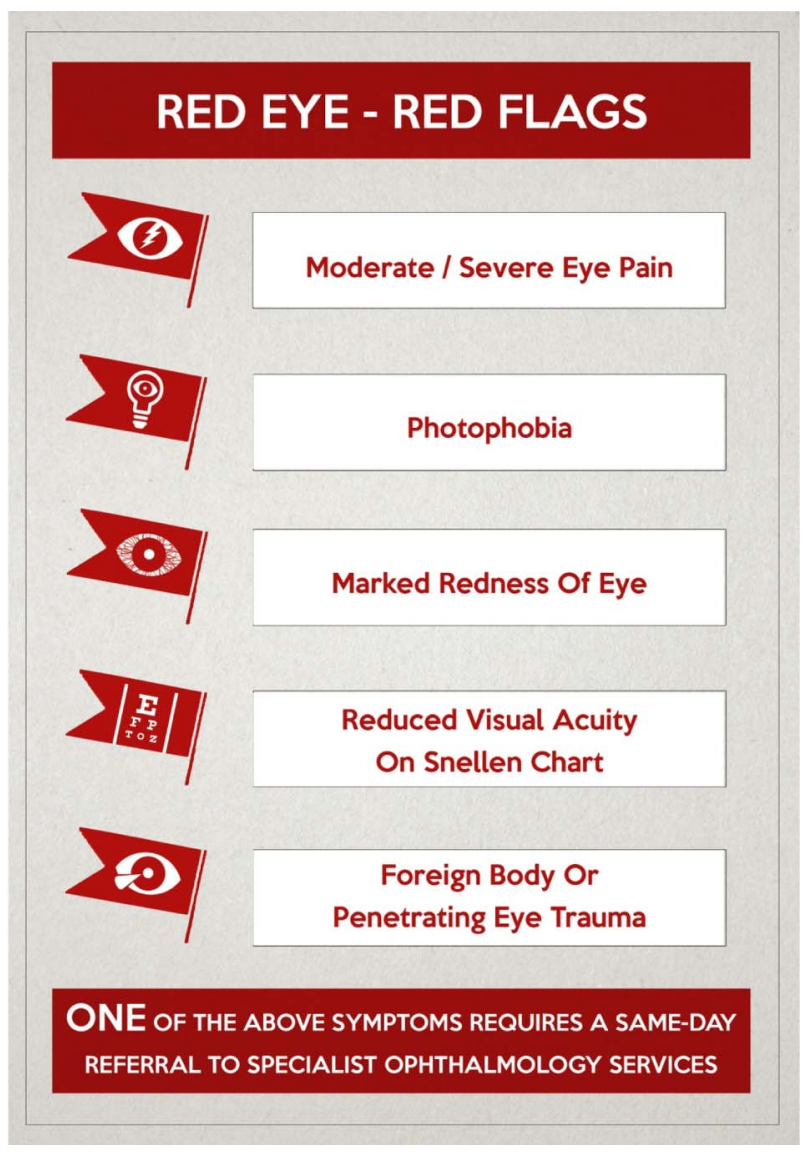

Figure 1. Red Flags poster, placed on the pin-board in every consultation room 


\section{A GUIDE TO THE SNELLEN CHART}

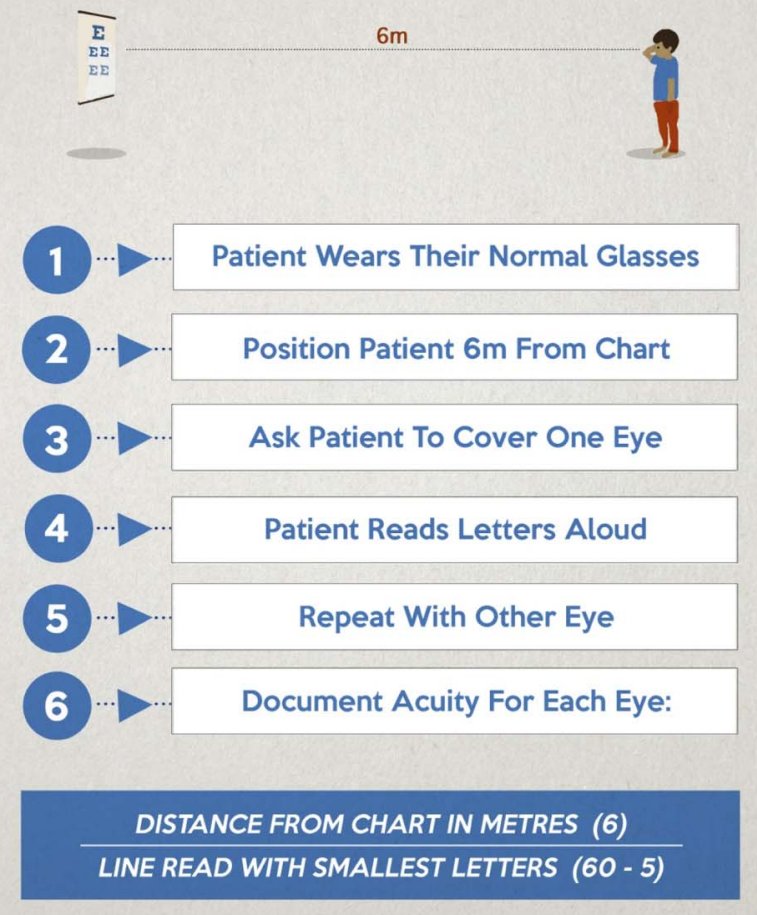

Figure 2. A Guide to The Snellen Chart poster, available in the red eye survival kit \& placed on the pin-board in every consultation room

teaching session was delivered. Following the teaching session, barriers to red-flag assessment were discussed.

The three main themes to emerge from the meeting were:

1. Appointment time-limitations

2. Lack of experience with eye assessment and eye conditions

3. The misconception that an assessment for red-flag features required specialist equipment.

These factors were addressed through further discussions on each topic and interactive demonstrations of red-flag feature assessments.

It was thought that the teaching session on visual acuity measurement would encourage use of the practice Snellen-chart, which was located along a corridor, adjacent to the waiting room. Feedback from clinicians demonstrated that they were unwilling to utilise the chart due to time-constraints on appointments, particularly with less mobile patients, and requested an alternative method of assessing visual acuity.

Outcome measurements were collected after eight weeks, meeting the same criteria as the baseline measurements, in order to accurately compare results. The sample size was smaller than expected (eight consultations) so the authors decided to increase the length of the second cycle to sixteen weeks.
Cycle 2:

Email correspondence provided further feedback on the barriers to red-flag assessment. One emerging theme was the need for permanent visible reminders of the red-flag features and referral criteria, as well as the previously mentioned alternative assessment modality for visual acuity. Based on this feedback, each consultation room was then provided with a 'red eye survival toolkit'; a folder containing:

1. A three-metre Snellen-chart (figure 3).

2. A guide to the Snellen-chart poster (figure 3).

3. A three-metre long tape measure (figure 3).

4. An illustrated guide to eye-drop application (figure 3).

It was agreed that each doctor was going to place the Snellen-chart provided on the wall within the consultation room, and measure out three metres in a convenient location.

Along with the toolkit, copies of the illustrated red-flag posters presented in the original teaching session were pinned onto the pin-board in every consultation room, immediately visible to each GP from their desk.

Inclusion of further tools in the red eye survival kit were considered and discussed. These included dilating eye-drops, ophthalmoscopes, pin-holes, and fluorescin staining drops. Each GP already possessed a pen-torch to assess for photophobia and pupil reflexes. Considering that time was a major barrier, and the red-flag features could be assessed through history and examination alone, these additional tools were considered inefficient and non-sustainable.

A template for the consultation software (figure 4), was discussed as a further intervention. The intention was that it would appear when a consultation was coded 'red eye', in a similar fashion to the existing template for laterality when any eye condition was coded. The template included visual prompts and space to document the assessment of each red-flag feature, laterality of the affected eye, and a reminder to refer to ophthalmology if a red-flag feature was present. Following long discussions, the majority of the clinicians felt that this intervention was unnecessary, and it was not implemented.

Further retrospective data collection took place after sixteen weeks as planed (24 weeks after the baseline data collection). The parameters measured met the same criteria as those in the baseline, and cycle 1 measurement.

\section{RESULTS}

Cycle 1:

Eight weeks following the teaching session, data was collected from eight red eye consultations, meeting the same criteria as the baseline data collection.

Visual acuity was formally measured $37.5 \%$ (3/8) of the time. This was a significant increase from baseline 
Figure 3. 'Red eye survival kit' containing: (1) a three-metre Snellen chart, (2) a guide to the Snellen chart poster, (3) a three-metre tape measure, and (4) an illustrated guide to applying eye-drops

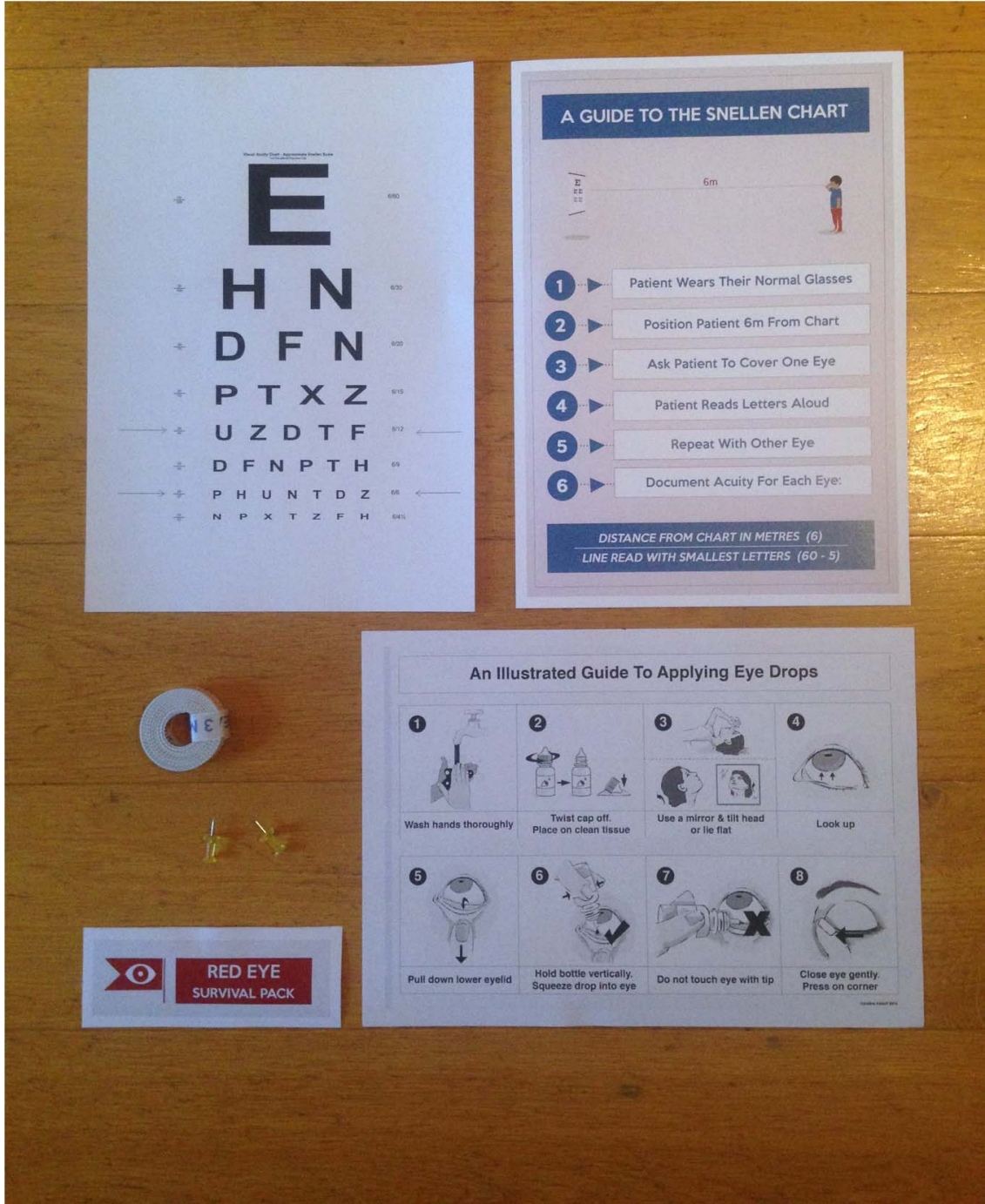

$(\mathrm{p}=0.0292)$. Ocular pain was assessed in $75.0 \%(6 / 8)$ of consultations. However only $25.0 \%$ (2/8) of assessments involved quantification of the severity of the pain; which was not significantly different from baseline $(\mathrm{p}=$ $0.6755)$. Redness of the eye was examined in $100 \%(8 / 8)$ consultations, which was a hopeful sign, but the extent of the redness was only documented in $37.5 \%$ (3/8) assessments, this increase from baseline was not considered significant $(p=0.1107)$. Photophobia was assessed $50.0 \%$ $(4 / 8)$ of the time, this was a significant increase $(\mathrm{p}=$ $0.0248)$. Laterality of the eye was documented $100.0 \%$ $(8 / 8)$ of the time; this was unchanged from baseline.

The mean number of red-flag features assessed in each consultation increased from 0.9 at baseline to 1.5 after one cycle. This increase was not significant $(\mathrm{p}=0.064)$.

Three patients presented with red-flag features; of these $33.3 \%$ (1/3) patient was appropriately referred to same-day ophthalmology services, a decrease from baseline, but not significant $(\mathrm{p}=0.7662)$.

Cycle 2:

The third set of outcome measurements were collected after a further sixteen weeks, from 27 red eye consultations. The results demonstrated a significant improvement in almost every parameter from the original data.

Pain was documented and quantified in $81.5 \%$ (22/ 27 ) of consultations. This was a significant improvement from baseline $(p=0.005)$. Similarly, redness of the affected eye was quantified in $66.7 \%$ (18/27) of consultations, also demonstrating a significant improvement $(\mathrm{p}<0.001)$. Visual acuity, using the Snellen-chart was documented in $55.6 \%(15 / 27)$ of consultations, this also showed significant improvement $(\mathrm{p}<0.001)$. Assessment of foreign body/penetrating eye injury was performed in $51.8 \%(14 / 27)$ of consultations, another significant increase $(\mathrm{p}<0.001)$.

The only red-flag to remain consistently poorly documented was photophobia, despite being one of the more straightforward red-flag features to assess, and particularly important to exclude. Photophobia was only assessed in $18.5 \%(5 / 27)$ of consultations, a small increase from baseline $(\mathrm{p}=0.75)$.

Every patient had at least one red-flag feature assessed; most two to four. The mean number of red-flag features assessed in each consultation rose from 0.9 at baseline 


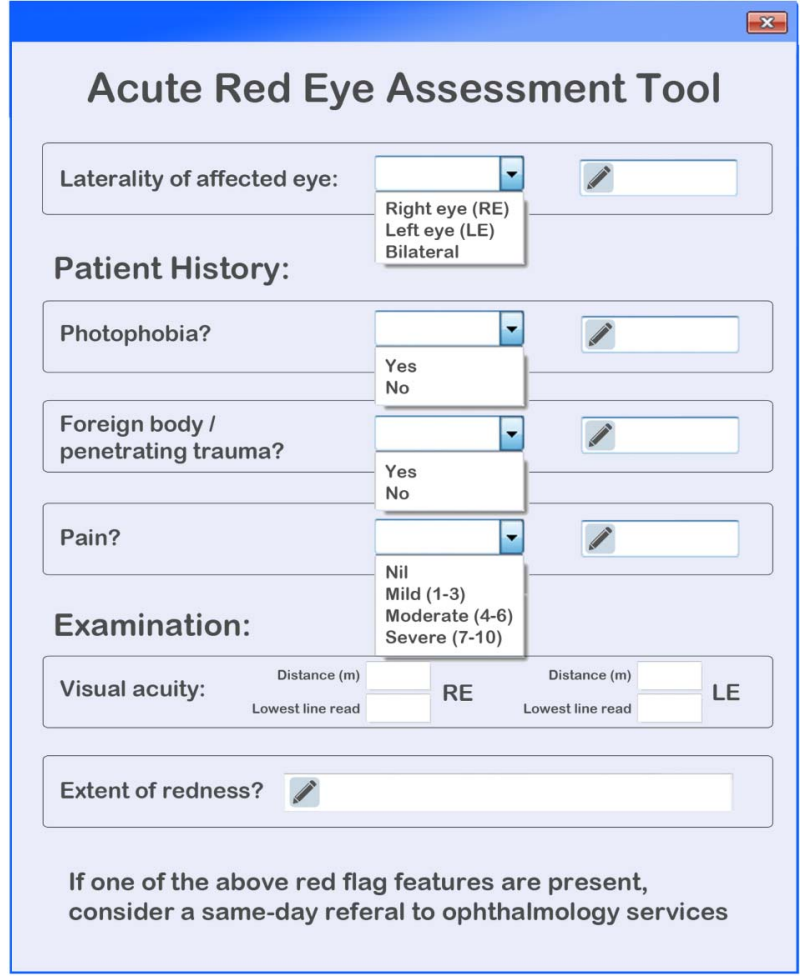

Figure 4. A prototype of a red eye assessment tool template proposed to install on the consultation software

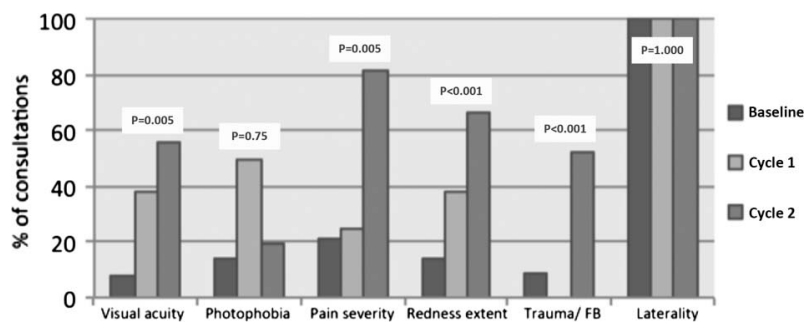

Chart 1 Percentage of consultations in which each red-flag feature was assessed $P$-values represent statistical significance between baseline values and values of data collected after two improvement cycles (at 24 weeks)

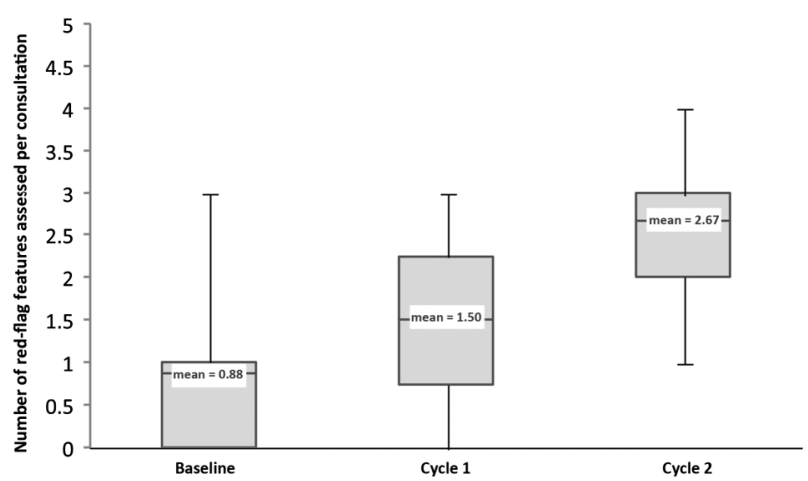

Chart 2 Mean number of red-flag features assessed per red eye consultation $P$-values represent statistical significance between baseline \& improvement cycle 1values (collected after 8 weeks), and baseline \& improvement cycle 2 values (collected after 24 weeks)

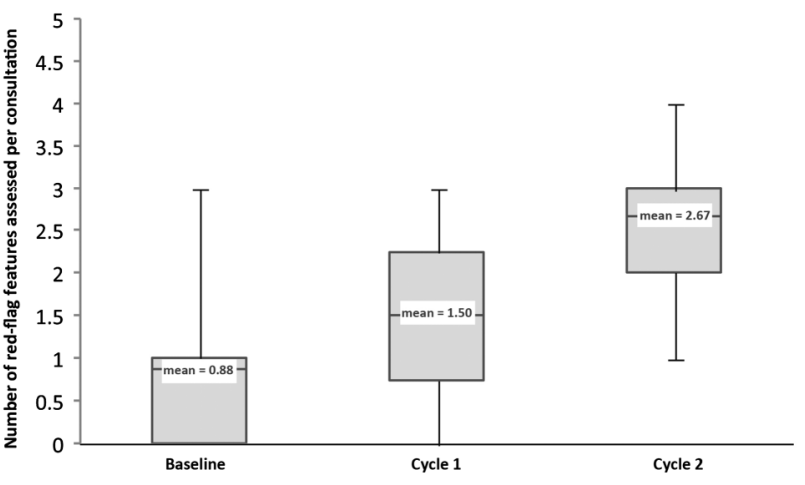

Chart 3 Percentage of patients exhibiting red-flag features who were appropriately referred to same-day ophthalmology services $P$-values represent statistical significance between baseline \& improvement cycle 1 values (collected after 8 weeks), and baseline \& improvement cycle 2 values (collected after 24 weeks)

to 2.7 after two improvement cycles. This was a significant increase $(\mathrm{p}<0.001)$.

Of the consultations, $29.6 \%(8 / 27)$ of patients demonstrated a red-flag feature, and of these patients $75.0 \%$ $(6 / 8)$ were appropriately referred to same-day ophthalmology services. Despite a large increase from baseline data, the numbers involved were too small to determine significance $(p=0.1015)$.

\section{LESSONS AND LIMITATIONS}

This project demonstrated that improvements in primary care eye assessments can be achieved through effective teaching sessions. The results reinforced the importance of continuing medical-education, and lifelong-learning in the medical profession.

Interventions need to be designed with the service-user in mind in order to succeed. In this project the interventions were formulated from discussions with the practice staff, and could be tailored to meet their needs. For example the provision of the three-metre Snellen-charts to each consultation room was the result of staff feedback on the impracticality of the existing practice chart.

Another strength of this project was the sustainability of the intervention design. The posters provided as part of the toolkit were still in situ at six months, as were the toolkits themselves. Furthermore, the toolkits will not expire or need replacement. The production of all of the toolkits cost $\hat{A} £ 8$ as a one-off payment and photocopies of the posters can be made at 50 pence for a colour copy. Email correspondence (figure 5) is used to send annual reminders describing the red-flag features, most appropriate method of assessment, and referral criteria to all staff at the practice at no extra cost.Despite these considerations, there were only two data collection points, spanning a six month period. Further data collection is required in order to determine the true sustainability of these interventions. 
Figure 5. Contents of annual email-reminder sent to all staff at the health-centre. The email contained details about the red-flag features, methods of assessment and the referral criteria

\author{
Re: Red eye Audit \\ 1 message \\ Kilduff, Caroline \\ $\mathrm{Hi}$ \\ I'm just emailing you an annual reminder of the red-flag features to assess in patients \\ presenting with a red eye \\ Red-Flag features: \\ 1.Moderate to severe pain (pain 5/10 and above) - assess through history \\ 2. Marked redness of affected eye (unilateral red eye more likely to be serious than bilateral red \\ eyes) - assessed via eye inspection \\ 3. Photophobia - assessed via history question or shining pen-torch in patient's eye \\ "* (if shining light- it's a good opportunity to check for pupillary reflexes- pupils should be equal \\ size and reactive- if not refer to ophthalmology) \\ 4. Reduction in visual acuity (VA) - each consultation room is equipped with a $3 \mathrm{~m}$ Snellen chart \\ in the red eye survival kit. \\ "Anyone with a smart phone- there is a great free app called "Snellen Chart" (it has a yellow \\ background) - hold your phone at $1 \mathrm{~m}$ and assess VA as per instructions (whatever line the \\ patient reads down to corresponds with the equivalent VA at $6 \mathrm{~m}$ )-very fast!! \\ 5. Foreign body or penetrating eye trauma - patient history and eye inspection if obvious \\ Then... \\ If any one of the above red-flag features are present, please refer the patient to same-day \\ ophthalmology services as these features can indicate a sight-threatening condition. \\ Thanks! \\ BW \\ Caroline and Charis
}

The main challenge faced was the implementation of the consultation software template. The rationale behind the template was to reduce the cognitive-load placed on the clinician, by reminding them of the required assessments to perform, and also inform them of the appropriate referral criteria.This was rejected as the staff felt that there were too many templates and pop-ups, and these can become distracting. The printed posters were considered an adequate visual reminder of assessment content and referral criteria.

One limitation to this project was the fact that the quality improvement team were medical students on rotation, prior to final examinations, with the next team arriving six months later, preventing a handover of information. Mostly, interventions are more sustainable when a handover occurs between incoming and outgoing teams.

Another limitation of this project was the small sample sizes in both improvement cycles. This was due to a short follow-up period (due to the authors' foundation jobs increasing geographical distance from the practice) and the reduction in red eye consultations during the summer months. This impacted on the accuracy of the results, due to the increased margin of error, and reduction in power to detect significant differences between data-sets.

Progress measurements were considered during cycle 1. These included time taken per red eye consultation, and GP confidence in assessment and management of red eye conditions. Due to the retrospective nature of the data collection, it was not possible to measure consultation timings. It was considered that filling in confidence questionnaires would increase time spent on paperwork, so these measurements were not taken.

The interventions in this project were not piloted before implementation. If the project were to be repeated, it would be best to select a single GP, deliver the intervention, and collect data from a small number of their subsequent red eye consultations. This would inform further intervention development before implementation on a large scale.

A similar project was noted to have taken place in 2014. Although unknown to the authors during the audit process, it demonstrated similar improvements in red-flag assessments following similar interventions. ${ }^{17}$ Our project built on the findings from the previous project by incorporating a teaching session, and permanent visual aids. The time to re-audit was extended from six to 24 weeks, and data on referral to ophthalmology services was included in the audit process.

\section{CONCLUSION}

This project reflected the literature in highlighting low confidence amongst GPs when dealing with red eye 
conditions. Rates of completed eye assessments were very low, and the content of consultations varied, following no fixed pattern, often missing key elements.

Barriers to assessment were identified during the practice meeting, and included time-constraints on appointments, inexperience performing ocular examinations, and lack of awareness of the red-flag features. A teaching presentation, and supporting posters provided to each consultation room, improved the quality of red eye assessments, as well as the rates of appropriate referral.

Despite the high turnover of staff in primary care, the project interventions were considered sustainable, due to the longevity of the posters provided in each consultation room. These would not need replacement unless the NICE CKS advice was updated. Additionally, the authors continue to maintain correspondence with the staff in the practice via email, despite geographical distance.

These results are generalisable throughout primary healthcare settings, as demonstrated by the similarity in outcome measurements between this project and that performed by Tei in 2014. ${ }^{17}$ A further identical project is planned for another primary care practice during a community placement in 2017.

This project emphasised the need for training in nonophthalmic medical fields to be improved. There is an opportunity for a cross-specialty transfer of knowledge, which would benefit all involved.

Next steps:

The results have initiated an interest in improving undergraduate ophthalmology education. A local teaching course was run in 2015 for fourth-year medical students at one university. The aim was to improve familiarity with eye assessments from an early stage. The course objectives included practical ophthalmic assessment, recognition and management of common eye conditions, recognition of red-flag features and sight-threatening conditions, and effective communication of examination findings. Based on good feedback, this course is to be repeated regionally in July 2016.

Following a foundation placement on a trauma high dependency ward, a project is being set up to improve ophthalmic assessment in a trauma setting. Many trauma patients have ocular involvement or peri-orbital injuries, and ward-based doctors appear to face the same confidence and educational issues as their primary care colleagues.

Acknowledgements We would like to acknowledge Dr Faz Khwaja and the staff at the Chrisp Street Health Centre for their participation in this project.

\section{Declaration of interests Nothing to declare}

Ethical approval According to the policy activities that constitute research at the Medical Research Council, Health Research Authority, this work met criteria for operational improvement activities exempt from ethics review.

Open Access This is an open-access article distributed under the terms of the Creative Commons Attribution Non-commercial License, which permits use, distribution, and reproduction in any medium, provided the original work is properly cited, the use is non commercial and is otherwise in compliance with the license. See:

- http://creativecommons.org/licenses/by-nc/2.0/

- http://creativecommons.org/licenses/by-nc/2.0/legalcode

\section{REFERENCES}

1. McDonnell PJ. How Do General Practitioners Manage Eye Disease in the Community? Br J Ophthalmol. 1988;(72):733-6

2. Cronau $\mathrm{H}$, Kankanala RR, \& Mauger T. Diagnosis and Management of Red Eye in Primary Care. Am Fam Physician. 2010;(8):137-44

3. Wirbelauer C. Management Of The Red Eye For The Primary Care Physician. Am J Med. 2006;(119):302-306

4. Leibowitz H. The Red Eye. N Engl J Med. 2000;(343):345-351

5. National Institute of Clinical Excellence (revised 2012) Clinical Knowledge Summaries [Available online: http://cks.nice.org.uk/ red-eye]

6. Featherstone PI, James C, Hall MS, \& Williams A. Genera practitioners' confidence in diagnosing and managing eye conditions: a survey in south Devon. Br J Gen Prac. 1992; (42):21-24

7. Jackson CL. Misdiagnosis of Acute Eye Diseases by Primary Health Care Providers: Incidence and Implications. Med J Aust. 2009; (190):343-344

8. The Royal National Institute of the Blind (2013) Sight loss UK 2013 [Available online: https://www.rnib.org.uk/sites/default/files/Sight_ loss_UK_2013.pdf]

9. The Royal College of General Practitioners (revised 2015) Eye Health [Available online: http://www.rcgp.org.uk/eyehealth]

10. Wilson A. The Red Eye: A General Practice Survey. J R Coll Gen Pract. 1987;(37):62-64

11. Roy F. The red eye. Ann Ophthalmol. 2006;(38):35-38

12. Graber M, Gordon R \& Franklin N. Reducing Diagnostic Errors in Medicine: What's the Goal? Acad Med. 2002 (77):981-2

13. Baylis O, Murray $P$ \& Dayan M. Undergraduate Ophthalmology Education - A Survey of UK Medical Schools. Med Teach. 2011 (33):468-71

14. Mahmood A. \& Narang A. Diagnosis and Management of The Acute Red Eye. Emergency Medicine Clinics. 2008. (26):35-55

15. Shields T. \& Sloane P. A comparison of eye problems in primary care and ophthalmology practices. Fam Med. 1991 (23):544-546

16. Foster A. Red eye: the role of primary care. Comm Eye Health 2005;(18):69-72

17. Teo MAI. Improving acute eye consultations in general practice: a practical approach. BMJ Qual Improv Report. 2014;(3):1 